\title{
SLIDING MODE CONTROL DESIGN FOR HAPTIC SYSTEM WITH VIBRATION MODE
}

\author{
Naveen Kumar \\ Dept. of Electrical Engineering \\ National Institute of Technology, Kurukshetra \\ Kurukshetra, India \\ naveenvermaindia@gmail.com
}

\author{
Jyoti Ohri \\ Dept. of Electrical Engineering \\ National Institute of Technology, Kurukshetra \\ Kurukshetra, India \\ ohrijyoti@rediffmail.com
}

Article history:

Received 11.07.2020, Accepted 10.12.2020

\begin{abstract}
Haptic is cutting edge technology having vast application mobile, home, robotics and tele-operations etc. It has enormous scope for tele-surgery using robotic arm with haptic feedback. It brings various dicsipline such as biomedical, neurological, physics and engineering on a single platform. It have numerious scope for all field. For this, haptic robotic arm should able to emulate the desired trajectory with minimum disturbances. In the real time, a robotic haptic arm have various uncertainty, vibrations and other unmodelled parameters which impact the output adversely. In this paper, a haptic device has been modelled in presence of internal vibration mode. A robust nonlinear sliding mode control (SMC) has been proposed in this paper. The efficacy of designed controller has been verified using simulation experiment. It has been observed that using the proposed method system follows desired trajectory accurately.
\end{abstract}

\section{Key words}

Haptic system, vibration mode, SMC, tracking, controller design.

\section{Introduction}

Haptic is one of this most demanding technology having wide application in video games, pilot training, and tele-surgical operations. Unlike the conventional robotic system, it allows user to interact with virtual or a remote environment with the sense of touch. With this mechanism, both user and the device inhabit the same workspace. Therefore, stability becomes an important factor for the user. The critical stability of haptic system depends on many factors such as uncertainty, delay, internal vibration modes, human operator etc. [Kumar and Ohri, 2020c] In literature, various author have worked on the stability of haptic system and designed the suitable controller to address these issues. In [Gil et al.,
2004] Gil et. al. have proposed the routh Hurwitz criterion for stability one dof haptic system. In [Dang et al., 2012] Dang et al have proposed LMI based stability approach for haptic system. Some authors has proposed conventional as well as optimal control strategy to address the stability issue [Kumar and Ohri, 2015]. In [Patrascu and Ion, 2016] uses genetic algorithm, FLC control design [Bashiti et al., 2018; Kumar and Ohri, 2017], and SMV has been used in [Kumar and Ohri, 2020b], estimation control method for nonlinear system in [Filippova, 2017], vibration method in [Tomchina, 2019] and LMI based method proposed in [Saragih, 2018]. In these articles, author have proposed the control strategy considering the delay and other uncertainty parameters. Further, the addition of internal vibration mode in the haptic system is proposed to enhance the work ability in real environments [Diaz and Gil, 2009]. In this paper, internal vibration mode has been considered into the haptic system. The addition of vibration into the system make the system nonlinear in nature. The conventional and optimal control strategy proposed in literature found unsuitable in this system model. So, a robust nonlinear Sliding mode control (SMC) has been proposed in this paper to ensure the stability and minimization in tracking he desired trajectory for haptic system. The organization of this paper is as follow: Section 2 contain the problem formulation, section 3 explains the SMC based control strategy. The section 4 contains the simulation experiment and result followed by result section.

\section{Problem Formulation}

\subsection{Perfectly Rigid Haptic Device}

A haptic device modelling shown in Fig. 1 is perfectly rigid model which is commonly used for haptic system description in literature. This model contains mass ' $m$ ', viscous damping ' $\mathrm{b}$ '. An input force ' $F_{r}$ ' is exerted by 
means of motor and input force by the user is ' $F_{u}$ '. So a single transfer function defined for this model [Diaz and Gil, 2009; Kumar and Ohri, 2020a] is (1).

$$
G(s)=\frac{x}{F_{d}+F_{u}}=\frac{1}{m s^{2}+b s}
$$

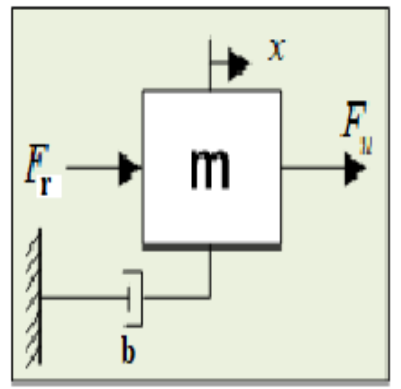

Figure 1. Spring damper model of haptic system

Further, the elastic deformation are generated in the haptic device between the user and driving motor which result change in output and referred as vibration mode in literature [Ciaurriz et al., 2014; Dang et al., 2012]. This vibration mode is included into the system which makes system nonlinear, as shown in Fig. 2.

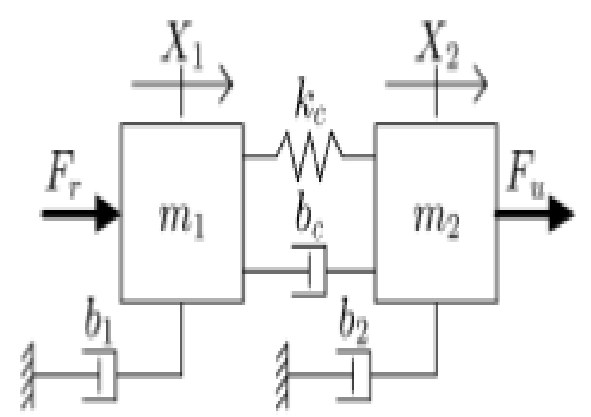

Figure 2. Haptic system with internal vibration mode [Diaz and Gil, 2009]

Here, the interface of system is combination of two masses connected by link mass ' $m_{1}$ ' and force exerted by on this by the motor itself, other is device mass ' $m_{2}$ ' the force exerted on this by the user. These masses are connected by spring and a damper shown in $\left(k_{c}\right.$ and $\left.b_{c}\right)$ Fig. 2. Due to vibration mode, physical interpretation of masses changes. The complete dynamics of system with vibration mode shown in Fig. 2 is given in (2).

$$
x=\left[\begin{array}{l}
x_{2} \\
x_{1}
\end{array}\right]=\left[\begin{array}{ll}
G_{2}(s) & G_{c}(s) \\
G_{c}(s) & G_{1}(s)
\end{array}\right]=G_{f}
$$

Here $\mathrm{G}$ is the transfer function of the hapic system with vibration mode is as given in (3) [Gil et al., 2004]

$$
G=\frac{1}{p(s)}\left[\begin{array}{cc}
p_{1}(s) & k_{c}+b_{c} s \\
k_{c}+b_{c} s & p_{2}(s)
\end{array}\right]
$$

where

$$
\begin{aligned}
& p_{1}(s)=m_{1} s^{2}+\left(b_{1}+b_{c}\right) s+k_{c} \\
& p_{2}(s)=m_{2} s^{2}+\left(b_{2}+b_{c}\right) s+k_{c} \\
& p(s)=p_{1}(s) p_{2}(s)-\left(k_{c}+b_{c} s\right)^{2}
\end{aligned}
$$

Using the critical stiffness analysis of system transfer function found in (Diaz Gil, 2009), the $G_{1}(s)$ is selected as relevant transfer function.

The physical parameters of haptic system for PHANToM haptic device are as given in Table: 1 [Diaz and Gil, 2009].

Table 1. Physical parameters for PHANTom 1.0 haptic device

\begin{tabular}{c|c|c}
\hline Parameters & Variable & Without user values \\
\hline \hline Device mass & $\mathrm{m}$ & $1.05 \mathrm{gm}^{2}$ \\
Device damping & $\mathrm{b}$ & $0.0085 \mathrm{Nms} / \mathrm{rad}$ \\
Device mass 1 & $m_{1}$ & $0.895 \mathrm{gm}^{2}$ \\
Device damping 1 & $b_{1}$ & $0.0085 \mathrm{Nms} / \mathrm{rad}$ \\
Connection damping & $b_{c}$ & $0.0057 \mathrm{Nms} / \mathrm{rad}$ \\
Connection stiffness & $k_{c}$ & $18.13 \mathrm{Nms} / \mathrm{rad}$ \\
Device mass 2 & $m_{2}$ & $0.155 \mathrm{gm}^{2}$ \\
Device damping 2 & $b_{2}$ & $0 \mathrm{Nms} / \mathrm{rad}$ \\
\hline
\end{tabular}

\section{SMC Model Design}

The Haptic system has become the nonlinear after adding the effect of single vibration mode. Therefore linear controller used in [Kumar and Ohri, 2015; Kumar and Ohri, 2020a], could not be employed to control the position and force after addition of nonlinearity. SMC has given promising results while dealing with nonlinear systems in presence of various uncertainty. It is known for high speed switching control to track the nonlinear plant trajectory on a user defined surface in the state space called sliding surface $s(x)$. It further maintain the plant state trajectory to this surface for the consequent time period. It maintain stability and dynamic performance of the system [Tirandaz, 2018]. 


\subsection{Reaching Law for Sliding Mode}

Sliding surface reaching law is divided into two phases: reaching phase and sliding phase. In first phase, the non-zero initial states of system is restricted to reach the sliding surface. In next phase, system states trajectory are forced to remains on the surface till equilibrium point is reached as shown in Fig. 3.

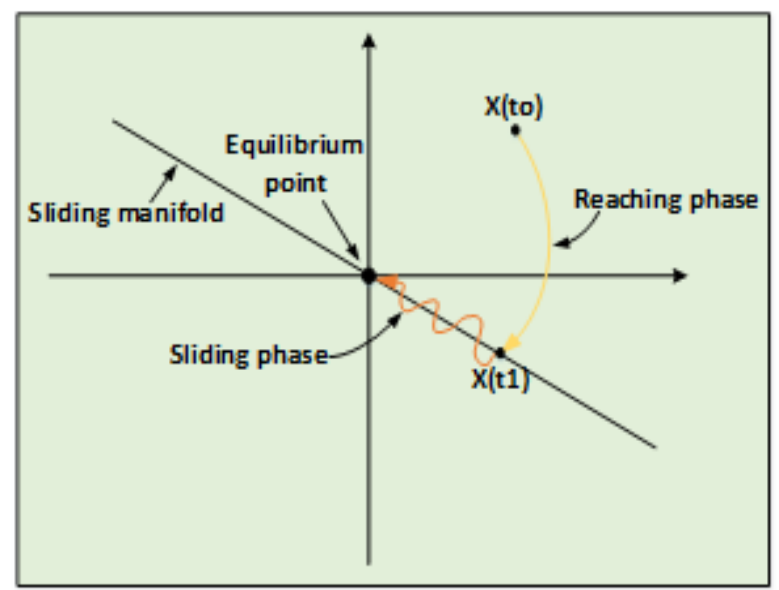

Figure 3. Sliding mode control step of operation

\subsection{First Order Sliding Mode Control}

The first order SMC gives better control than classical control techniques. In first order SMC, the sliding surface which depends upon the trajectory. The function $\mathrm{s}$ should be selected in such a way that it forces $s=0$. It gives rise to a stable differential equation and solution $\mathrm{e}$ of which will tend to zero eventually. The sliding surface is mainly a linear combination of the following type:

$$
s=\dot{e}+\lambda e
$$

where $\lambda=\operatorname{diag}\left(\lambda_{1}, \lambda_{2}, \ldots, \lambda_{n}\right)$ is a diagonal positive definite matrix.

First order sliding mode control is discontinuous across the surface $s=0$ and is given below:

$$
u=-K \operatorname{sign}(s)
$$

where $K=\operatorname{diag}\left(K_{1}, K_{2}, \ldots, K_{n}\right)$ is a diagonal positive definite matrix in which $K_{i}$ is positive constant

Where, $e \epsilon R^{3 * 1}$ error matrix, which can be consider as $e=\theta-\theta^{d}, s \in R^{3 * 1}, \theta$ represents actual trajectory and $\theta^{d}$ represents the desired trajectory

$$
\operatorname{sign}(s)= \begin{cases}1, & \text { if } s>0 \\ 0, & \text { if } s=0 \\ -1, & \text { if } s<0\end{cases}
$$

A damping function is introduced in the conventional SMC to damp the transient behavior in tracking control. Conventional SMC with damping can be given as

$$
u=-K \operatorname{sign}(s)-A s
$$

where $K, \lambda$ and $\mathrm{A}$ are the gain matrices which are defined as below

$K=\operatorname{diag}\left(K_{1}, K_{2}, K_{3}\right)$ is a diagonal positive definite matrix

$\lambda=\operatorname{diag}\left(\lambda_{1}, \lambda_{2}, \lambda_{3}\right)$ is a diagonal positive definite matrix

$A=\operatorname{diag}\left(A_{1}, A_{2}, A_{3}\right)$ is a diagonal positive definite matrix

\section{Simulations and Results}

The simulation has been performed for haptic system with first internal vibration mode. The SMC has been applied to 1-DOF phantom haptic system to find trajectory for haptic system as shown inFig. 4. The experiment has been performed on Intel i5 processor built-in personal computer with 4GB RAM. The simulation model.

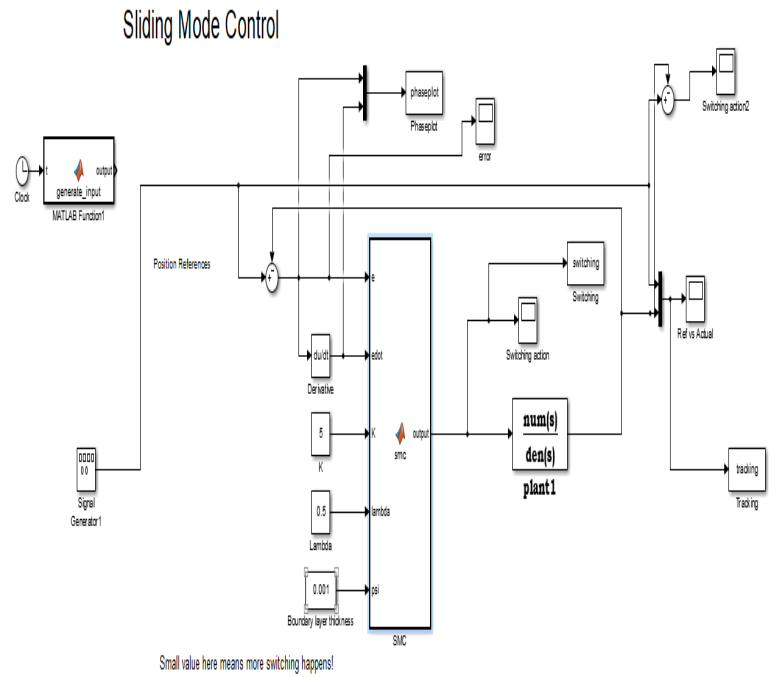

Figure 4. Simulation experiment model for haptic system with vibration mode

The transfer function model for haptic system with vibration mode is given in (8) using equation (3) and parameters given in Table: 1 .

$$
G_{1}(s)=\frac{0.95 s^{2}+0.0084 s+18.11}{0.7695 s^{3}+0.0689 s^{2}+1.0323 s}
$$

Using this, the simulation has been performed and corresponding results are shown in following figures. 


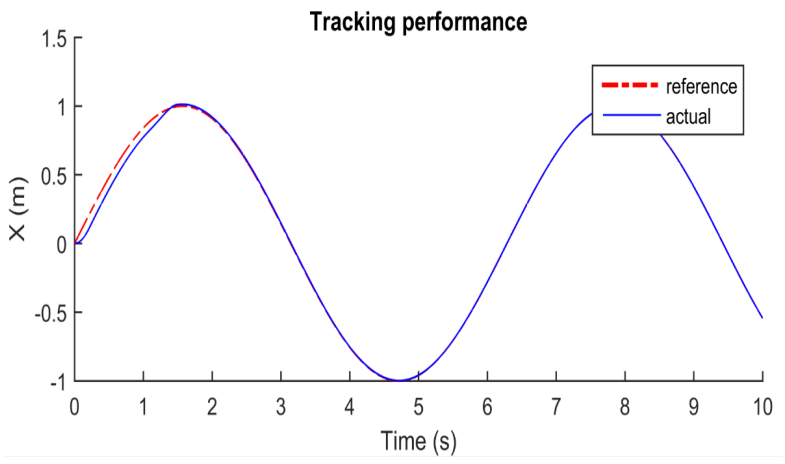

Figure 5. Trajectory tracking of haptic system with vibration mode

It has been observed from Fig. 5 that using SMC design, the system with vibration mode tracks the desired trajectory with upmost efficiency. The corresponding error plot is shown in Fig. 6.

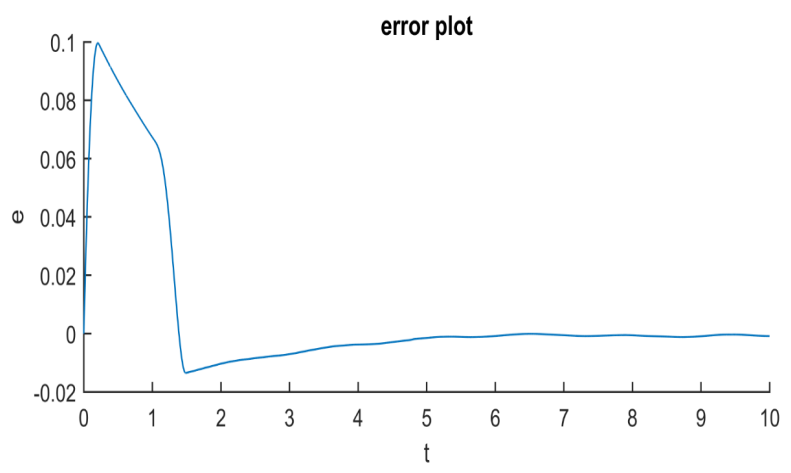

Figure 6. Error between desired and actual trajectory

The error trajectory in Fig. 6 shows that there is minimum between desired and actual trajectory. The switching action for designed SMC is shown in Fig. 7.

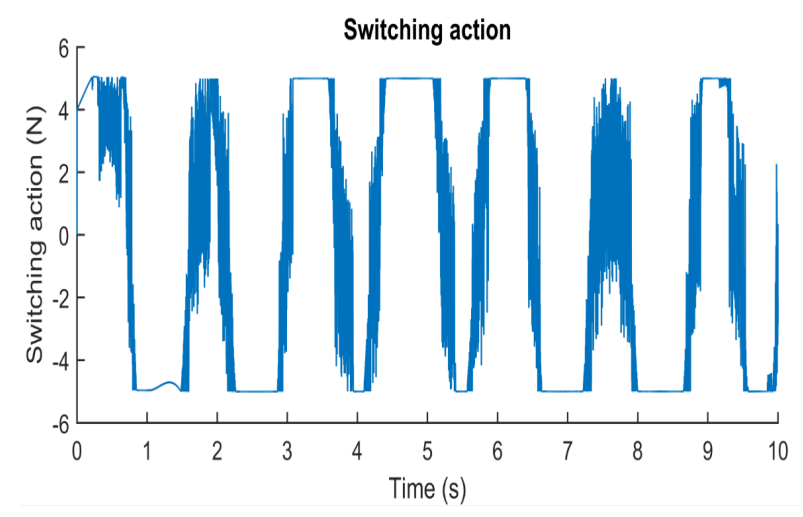

Figure 7. SMC switching action of control signal $(\tau)$ for haptic system with vibration mode
It has been observed from the above figures that using SMC, there is minimum error between desired and actual trajectory. It means, when user applies some force or input to the haptic manipulating device or haptic system, the same trajectory has been followed the system with minimum error. The switching action represents the motion of actuator to track and control the manipulator. It should be minimum. But minimizing the switching action will hamper the desired tracking. So for adequate performance of whole system, there must balance between the switching action and the trajectory tracking. Here Fig. 7 shows the adequate performance of control action SMC controller in presence of vibration mode in haptic system.

\section{Conclusion}

In this paper, haptic system with internal vibration mode has been presented. The modeling of haptic system with vibration mode makes system nonlinear and hence conventional and optimal control strategies are not applicable. So, a robust nonlinear SMC controller has been proposed in this paper. The performance of the designed controller has been observed. The system here tracks the trajectory with upmost efficiency. The error between the desired and actual tracking is very less. The switching action control signal is also gives satisfactory performance

\section{Acknowledgements}

The first author is thankful to the University Grant Commission (UGC), Delhi (India), for providing financial support to this work.

\section{References}

Bashiti, A., Malkawi, M., Khasawneh, M. A., and Murad, O. (2018). A novel neuro-fuzzy model to detect human emotions using different set of vital factors with performance index measure. Journal of Communications Software and Systems, 14 (1), pp. 121-129.

Ciaurriz, P., Diaz, I., and Gil, J. J. (2014). Stable discrete-time impedances for haptic systems with vibration modes and delay. IEEE Transactions on Control Systems Technology, 22 (3), pp. 884-895.

Dang, Q. V., Vermeiren, L., Dequidt, A., and Dambrine, M. (2012). Analyzing stability of haptic interface using linear matrix inequality approach. In IEEE International Conference on Robotics and Biomimetics, ROBIO 2012 - Conference Digest, pp. 1129-1134.

Diaz, I. and Gil, J. J. (2009). Influence of Vibration Modes and Human Operator on the Stability of Haptic Rendering. IEEE Transactions on Robotics, 26(1), pp. 1-6. 
Filippova, T. F. (2017). Reachable sets of nonlinear control systems with state constraints and uncertainty and their estimates. Cybernetics and Physics, 6(4), pp. 185-194.

Gil, J. J., Avello, A., Rubio, a. A., and Flórez, J. (2004). Stability analysis of a 1 DOF haptic interface using the Routh-Hurwitz criterion. IEEE Transactions on Control Systems Technology, 12 (4), pp. 583-588.

Kumar, N. and Ohri, J. (2015). Design of Haptic Interface Controller under Noise Uncertainty and Delay Condition. Procedia Computer Science, 70, pp. 793 800.

Kumar, N. and Ohri, J. (2017). LabVIEW based fuzzy logic controller for haptic interface. In Proceeding of IEEE 8th International Conference on Computing, Communications and Networking Technologies, ICCCNT 2017, pp. 1-6.

Kumar, N. and Ohri, J. (2020a). Novel m-PSO optimized LQR control design for flexible link manipulator: An experimental validation. Majlesi Journal of Electrical Engineering, 14(2), pp. 81-92.
Kumar, N. and Ohri, J. (2020b). Novel m-PSO-SVM based Interface Controller Design for Haptic System. Journal of Communications Software and Systems, 16 (2), pp. 188-196.

Kumar, N. and Ohri, J. (2020c). Optimal interface controller design for haptic system. Cybernetics and Physics, 9(1), pp. 41-50.

Patrascu, M. and Ion, A. (2016). Evolutionary Modeling of Industrial Plants and Design of PID Controllers, vol. 40 of Studies in Systems, Decision and Control. Springer International Publishing, Cham.

Saragih, R. (2018). Bilinear control based on linear matrix inequalities. WSEAS Transactions on Mathematics, 17, pp. 352-358.

Tirandaz, H. (2018). Adaptive integral sliding mode control method for synchronization of supply chain system. WSEAS Transactions on Systems and Control, 13, pp. 54-62.

Tomchina, O. P. (2019). Control of vibrational field in a vibration unit: Influence of drive dynamics. Cybernetics and Physics, 8 (4), pp. 298-306. 